\title{
А.С. Лосев
}

\section{ОЦЕНКИ ПРЕДПРИНИМАТЕЛЬСКИХ СЕТЕЙ РЕГИОНАЛЬНОГО УРОВНЯ}

Рассматриваются показатели предпринимательских сетей, разработанные в работах М.Г. Светунькова и А.В. Волкова. Рассматривается методика использования современных математических алгоритмов обработки сетей, по уточнению оценок показателей предпринимательских сетей в предельных и промежуточных состояниях. В качестве основных показателей, предложенных М.Г. Светуньковым А.В. Волковым и рассмотренных в настоящей работе, выступают размерность сети, ее плотность связи, гетерогенность, ранг, степень централизации, степень мобильности структуры и степень структурной близости сети к сетеобразующему элементу. Более детальная проработка и оценка позволила объединить данные показатели и перейти от точечного анализа отдельных характеристик сети к системному анализу, формируя общую картину о структуре и функционировании исследуемой региональной сети. Число рассмотренных показателей оценки предпринимательской сети не ограничивается данным перечнем и может быть дополнено и расширено в зависимости от сферы деятельности. Уточненные показатели оценки предпринимательских сетей позволяют за счет использования современных математических методов перейти от описательного результата к детальному анализу, что говорит о более качественном анализе устройства и функционирования изучаемой сети.

Ключевые слова: предпринимательская сеть, региональная структура, оценки сети, показатели сети.

Стремительное развитие информационных и сетевых технологий отражается на всех сферах деятельности современного человека. В контексте столь агрессивного внедрения информационных технологий возникает естественная модернизация предприятий и стремление соответствовать, идти в ногу со временем. Наиболее интересным считается появление сетей различной природы (социальных, политических, предпринимательских, производственных и т.п.), как неотъемлемый фактор принадлежности к глобальной информационной сети, которая является платформой дальнейшего развития. В результате перехода на сетевое представление, широкое применение получил одноименный подход. С помощью сетевого подхода проводится анализ различных показателей и процессов, проходящих в исследуемых сетях. Особенную актуальность имеют задачи, возникающие в предпринимательских сетях: исследование феномена этнического предпринимательства, эффекта структурных пустот, изучение функционирования подобных сетей малых предприятий [1-6].

Актуальность исследования предпринимательских сетей обосновывается тем, что меняется восприятие рынка, следовательно, возникают новые законы развития и методы работы на нем. Рынок в данной концепции - это не просто сфера обмена благами или гибкая структура, ячейки которой заполняются

(c) Лосев А.С., 2017

Лосев Александр Сергеевич - канд. физ.-мат. наук, ст. научный сотрудник, Институт прикладной математики ДВО РАН, г. Владивосток, e-mail: A.S.Losev@yandex.ru. 
отдельными фирмами. Прежде всего, это сложный сигнальный механизм, который помогает фирмам выбрать и обустроить под себя определенные рыночные ниши в виде сетевых связей с поставщиками и потребителями, и эти ниши не существуют в готовом виде [1].

В отличие от предпринимательских организаций сети являются более устойчивыми и конкурентоспособными в соответствующем информационнотехнологическом пространстве современного рынка. Прямые контакты позволяют участникам сетей обмениваться информацией, осуществлять взаимоконтроль и оперативно разрешать конфликтные ситуации. Длительное знание друг друга помогает формировать социальный капитал в виде накопленных взаимных обязательств, формировать доверие и деловые репутации. Посредством сетей поддерживается конкурентное напряжение и одновременно оказывается взаимная поддержка, нацеленная на общую стабилизацию рынка. Сети позволяют также сформировать структуры представительства коллективных интересов. На их основе формируются ассоциации, лоббирующие интересы отдельных групп в структурах государственной власти. В итоге, контрактные отношения между участниками рынка дополняются социальными отношениями и, отчасти, замещаются ими, что не только не мешает, но и стимулирует получение взаимной выгоды от рыночного обмена [1-7].

В настоящей работе рассматриваются показатели предпринимательских сетей, разработанные М.Г. Светуньковым и А.В. Волковым [8]. С помощью современных математических методов обработки сетей получены уточненные оценки предложенных показателей в предельных случаях, а также рассмотрены промежуточные состояния сетей регионального уровня. Более детальная проработка и оценка позволила объединить данные показатели и перейти от точечного анализа отдельных характеристик сети к системному анализу, формируя общую картину о структуре и функционировании исследуемой сети.

Введем в рассмотрение предпринимательскую сеть, которая представляет собой коалиционное образование, не всегда однородное по конфигурации, составу или свойствам, но которое занимается хозяйственной деятельностью в соответствующем сегменте рынка. Она может быть представлена как связный ориентированный граф, узлами которых являются агенты, а дугами наличие связи между ними. Под связью между агентами будем подразумевать не только экономические трансакции, но и передачу информации, указаний и распоряжений. Следовательно, обратная связь будет характеризоваться как отчет о проделанной работе или возможность выйти напрямую на вышестоящего агента. Рассмотрение предпринимательской сети через ориентированный граф отличается от традиционного представления [9]. Однако наблюдения за работой предпринимательских сетей показывают, что неориентированный граф не в состоянии передать всю сложность производственных связей и удобен только при моделировании социальных сетей, основанных на паритетных началах без установления подчиненности. 
Ограничимся рассмотрением сетей небольшой размерности, не превышающих региональный уровень, что продиктовано рассматриваемыми показателями. Разработанные в работе [8] показатели и соответствующие оценки характеризуют предельные значения, описывая либо частные случаи, либо отдельные элементы сети. Более детальное их рассмотрение показало, что в случае сетей достаточно большой размерности данные показатели носят избирательный характер, а оценки не позволяют сформировать объективную картину.

Размерность сети (n) - показатель, характеризующий число агентов, из которых состоит сеть [8]. На первый взгляд, в чистом виде данный показатель не представляет интереса. Однако его значение напрямую отражается на всех последующих показателях, что не уменьшает его значимости и требует его отдельного рассмотрения. Помимо этого, если говорить о современных информационных технологиях, которые широко применяются и берутся на вооружение именно в предпринимательской сфере, то данный показатель может быть определяющим при их анализе, а именно: факт, что при организации связи в сети используется Интернет, приводит к тому, что данная сеть может носить не только корпоративный, но региональный и глобальный характер.

Плотность связи сети $(p)$ - отношение фактических связей в сети $\left(n_{\text {факт }}\right)$ к максимально возможному числу связей между всеми ее элементами [8]:

$$
p=\frac{n_{\text {факт }}}{n(n-1)} \text {. }
$$

В общем представлении предпринимательская сеть не может состоять меньше чем из двух агентов, также в ней не может быть изолированных агентов, иначе нарушается условие связности. Следовательно, можно утверждать, что сеть с минимальным числом ребер $n-1$ является жестко централизованной древовидной или радиальной [10].

Отсюда получаем, что плотность связи сети принадлежит интервалу $[1 / n ; 1]$. В случае $p=1 / n$ речь идет о прямом подчинении каждого агента вышестоящему, без обратной связи. В случае $p=1$ речь идет о полносвязной сети, состоящей из агентов одного уровня. Такую сеть можно сравнить с некоторым демократическим сообществом, решение в котором принимается голосованием или коалицией из универсальных агентов, где каждый может выполнять любую роль от исполнителя до начальника. Примером такой сети являются различные социальные сети, но не сети предпринимательского типа, так как они образуются на основе материальной выгоды, которая в большинстве случаев распределяется в соответствии с иерархией, отсутствующей в социальных сетях, основанных единомышленниками на паритетных началах.

Отдельно следует рассмотреть случай, когда $0,5 \leq p<1$, что говорит о достаточно высоком уровне организации сети с наличием обратных связей и делает 
сеть более гибкой. Наравне с этим при $p \rightarrow 1$ в сети образуются группы агентовуправляющих на различных уровнях сети, выстраивающей вертикаль власти, наличие которой в общем случае не противоречит единой централизации сети.

Степень цеентрализации сети - одна из величин, определение которой вызывает трудности, связанные с разногласиями в самом понимании данной характеристики. Интерпретация данной величины состоит в определении меры группирования агентов вокруг единого центра [11]. Для определения данной меры используют достаточно сложные формулы, основанные, как правило, на использовании показателей центральности актора с учетом нормирования числа связей $[12,13]$. В работе [8] авторам удалось получить более простую формулу, не предполагающую нормирования числа связей и расчетов показателей центральности акторов. Однако это упрощение свело значение степени централизации сети к вероятностной величине, характеризующей наличие или отсутствие центра в общем представлении. Использование данной величины в таком представлении не позволяет охарактеризовать сеть как содержащую один центр или множество.

Централизация - это концентрация прав принятия решений, сосредоточение властных полномочий на верхнем уровне руководства организации [14]. Следовательно, степень централизации сети должна определяться не только наличием или отсутствием управляющей верхушки, но и ее численностью, концентрацией и возможностью передачи решений на все уровни сети, т.е. отдаленностью самого низкого уровня сети от центра.

Расчет данного показателя является сравнительно сложной и трудоемкой задачей, однако разработанные методы в работе [15] для комплексного исследования ориентированных сетей позволяют уменьшить трудоемкость по сравнению с известными оценками степени централизации сети [11-13] и получить более точную оценку характеристики сети по сравнению с упрощенными методами [8]. Алгоритм «перечисления минимальных контролирующих наборов вершин в орграфе», полученный в работе [15], позволяет разбить сеть на кластеры, элементы которых образуют компоненты связности в ориентированном графе, порожденные исходной сетью.

Следовательно, можно утверждать, что каждый кластер, полученный в предпринимательской сети, образует совокупность агентов сети одного уровня. Помимо этого, в алгоритме из работы [15] на множестве кластеров определено отношение частичного порядка, которое однозначно определяет иерархию между ними. В случае с предпринимательской сетью иерархия образует вертикаль власти и строгое подчинение агентов в сети.

Таким образом, использование быстрых и компактных современных математических алгоритмов [15] позволяет преобразовать исходную сеть в сеть, состоящую из различных объединений агентов одного уровня власти. Размерность преобразованной сети можно трактовать как количество уровней 
иерархии в исходной сети. Кластеры, состоящие из одного агента, являются новыми элементами сети, которые еще не обросли связями. Кластер больше всех остальных частичного порядка однозначно определяется как управляющая верхушка, имеющая только исходящие связи и не находящиеся в подчинении, а количество выходящих связей данного кластера можно буквально считать как степень централизации сети.

Гетерогенность сети - показатель неоднородности элементов сети. Естественно предположить, что функционирующая предпринимательская сеть не должна состоять только из агентов экономической сферы. Следовательно, данный показатель определяет степень агентов, связанных с предпринимательской деятельностью, но не занимающихся ей напрямую. Речь идет о вспомогательных задачах, которые необходимо решать для поддержания функционирования любой сети и не только предпринимательской, например, работа с общественными организациями, политическими, государственными органами и т.д.

В качестве характеристики гетерогенности сети в работе [8] предлагается следующая величина:

$$
H=1-\frac{n_{\text {предпр }}}{n},
$$

т.е. единица минус отношение числа предпринимателей в сети к общему числу агентов сети. Данный показатель определяет процент гетерогенности сети, но мало информирует нас о расположении непредпринимательских агентов в сети. Необходимо отметить, положение агентов в сети играет огромную роль при оценке деятельности сети в целом. К примеру, сеть может содержать одного агента не своей сферы деятельности, но если он расположен в начале иерархии, то это одна ситуация, а если в конце, то это совершенно другая. В зависимости от этого можно не только говорить о законности и правомерности деятельности предпринимательской сети, но и предположить ее основные задачи и перспективы развития.

В целях уточнения расположения агентов, выполняющих вспомогательные задачи сети, предлагается рассмотреть их расположение в сети, полученной в результате кластеризации алгоритмом «перечисления минимальных контролирующих наборов вершин в орграфе». Логично предположить, что агенты, работающие над вспомогательными задачами, в данной сети будут представляться в виде кластеров, содержащих один элемент, замыкая на себе связи, выступая связующими элементами различных отделов с внешним миром или другими сетями. Также правомерно предположить, что агенты непредпринимательской природы не должны образовывать отдельные кластеры на верхних и средних уровнях иерархии сети, в противном случае возникает серьезное подозрение о легальности данной сети.

Следовательно, использование комбинированной оценки гетерогенности сети посредством оценки доли агентов непредпринимательской природы и их 
расположением в кластеризованном представлении сети позволяет более точно охарактеризовать природу деятельности сети, предположить о законности и некой стрессоустойчивости к воздействиям на нее извне.

Ранг сети - длина общей многоступенчатой связи, в которой один элемент сети связан с другими элементами [13]. В работе [8] авторы под рангом понимают среднее число связей, входящих в маршрут связи между двумя элементами сети по кратчайшему пути, которое определяется по формуле

$$
R=\frac{\sum_{i=1}^{n(n-1)} L(a-b)}{n(n-1)},
$$

где $L(a-b)$ - число связей в кратчайшей цепочке между агентами $a$ и $b$. Такое представление больше характеризует не ранг сети, а среднюю длину между любыми двумя агентами сети, которая мало интересна при анализе.

В качестве характеристики ранга предлагается использовать точный счет и оценить длину иерархической цепочки в кластеризованной сети, где каждый кластер находится в отношении частичного порядка. Отсюда следует, что количество уровней в сети, представленной через кластеры, и есть ранг.

Следовательно, ранг будет характеризовать необходимое число уровней, которое следует согласовать или пройти для установления связи между двумя агентами сети различных уровней. Данное представление ранга более обоснованно с позиции практики, так как вопрос об установлении количества связей, необходимых между двумя агентами одного кластера или одного уровня, не актуален и в большинстве случаев решается напрямую, что нельзя сказать о порядке согласований между отделами.

Степень мобильности структуры сети отражает изменение сложившихся связей между элементами с течением времени [8]. Предполагается, что структура сети является мобильной, если с течением времени происходит значительное изменение связей в рамках изучаемой сети: старые связи разрушаются вплоть до исключения элементов из сети, возникают новые связи между элементами, в том числе с включением в сеть новых элементов.

Определение степени мобильности сети требует ее рассмотрения во времени, т.е. необходимо рассмотреть матрицу смежности сети $\|x(t)\|_{i, j=1}^{n}$ в момент времени $t$ и матрицу смежности сети $\|x(t+1)\|_{i, j=1}^{n}$ в момент времени $t+1$. В работе [8] степень мобильности определяется следующим образом:

$$
M=\frac{\sum_{i=1}^{n} \sum_{j=1}^{n}\left(x_{i j}(t)-x_{i j}(t+1)\right)^{2}}{n(n-1)} .
$$


В зависимости от принимаемых значений из интервала [0;1], степень мобильности характеризует сеть либо абсолютно устойчивой при $M=0$, либо абсолютно мобильной при $M=1$. В случае промежуточного значения оценка носит относительный характер и не представляет большого интереса и практической значимости [8].

Данная мысль не всегда верна, так как именно промежуточный результат имеет больший интерес и практическую значимость. В этом случае предлагается рассмотреть матрицу смежности кластеров сети $\left\|x^{\prime}(t)\right\|_{i, j=1}^{k}$ в момент времени $t$ и матрицу смежности кластеров сети $\left\|x^{\prime}(t+1)\right\|_{i, j=1}^{m}$ в момент времени $t+1$, полученные в результате применения алгоритма «перечисления минимальных контролирующих наборов вершин в орграфе». В результате сравнения числа кластеров до $k$ и после $m$ при условии, что $M \in(0 ; 1)$, можно сделать следующие выводы:

1. Если $k<m$, то в сети произошло увеличение числа уровней или отделов за счет разбиения крупных отделов на более мелкие (при $M<0,5)$ или образования новых связей (при $M>0,5)$.

2 . Если $k>m$, то в сети произошло уменьшение числа уровней или отделов за счет укрупнения и объединения мелких (при $M>0,5)$ или прерывания связей и увольнения агентов (при $M<0,5$ ).

3. Если $k=m$, то все изменения в сети не повлияли на ее иерархию и носят локальный характер. К примеру, налаживание новых связей между агентами (при $M>0,5)$ или потеря старых, мало функционирующих связей между агентами (при $M<0,5$ ).

Данный алгоритм позволяет не просто численно оценить степень мобильности структуры сети, но и дать качественную оценку происходящих изменений в сети, которая более адаптирована к ее природе.

Степень структурной близости сети к сетеобразующему элементу может быть рассчитана в случае, если при изучении предпринимательской сети удастся установить наличие сетеобразующего элемента и однозначно его определить [8].

Определение данного показателя осложняется предварительной задачей по установлению факта присутствия сетеобразующего элемента, который не обладает четкими критериями. В частности, сетеобразующим элементом можно представить агента, обладающего наибольшим числом связей; фирму, обладающую наибольшим влиянием на рынке; структуру, способную с помощью административных или других методов воздействовать на рыночную ситуацию.

В работе [8] авторы предполагают, что этот элемент известен и степень структурной близости сети к сетеобразующему элементу определяется как числовая величина из промежутка (0;1]. Результат данной оценки носит вероятно- 
стный смысл и только в частном случае, при степени один, трактуется как непосредственная связь всех элементов сети с сетеобразующим элементом.

В данном случае в силу отсутствия формальных критериев поиска сетеобразующего элемента предлагается рассматривать его в зависимости от степени централизации сети. Степень централизации сети определяется посредством разбиения сети на кластеры и построения соответствующей иерархии. Следовательно, если речь идет о высокоцентрализованной сети, кластеризация которой представляется в виде дерева (четкая вертикаль управления) или радиальной сети (все кластеры в непосредственном подчинении выделенного элемента), то без сомнения сетеобразующим элементом будет выступать кластер агентов, определяющий управление всей сети. Исходя из этого степень структурной близости сети к сетеобразующему элементу может быть определена как высокая, а численная оценка будет равна рангу сети.

В случае низкоцентрализованной сети кластеризация будет содержать неявно выраженную иерархию с большим числом элементов на каждом уровне, что можно интерпретировать как отсутствие единого сетеобразующего элемента и степень структурной близости сети к сетеобразующему элементу можно считать низкой.

В случае децентрализованной сети в результате кластеризации будет получен один кластер, содержащий все элементы сети, следовательно, степень структурной близости сети к сетеобразующему элементу можно считать нулевой в силу отсутствия такого.

Таким образом, данный показатель носит индивидуальный характер и не может быть определен в каждой рассматриваемой предпринимательской сети, однако его отсутствие тоже можно считать соответствующей характеристикой сети и использовать при анализе.

В заключение следует отметить, что разработанные ранее показатели оценки предпринимательских сетей, рассмотренные в настоящей работе и уточненные за счет использования современных математических методов, позволяют перейти от описательного результата к системному анализу, что говорит о более качественном анализе устройства и функционирования изучаемой сети.

\section{Список литературы}

1. Радаев В.В. Рынок как переплетение социальных сетей // Российский журнал менеджмента. - 2008. - Т. 6, № 2. - С. 47-54.

2. Асаул А.Н., Скуматов Е.Г., Локтеева Г.Е. Методологические аспекты формирования и развития предпринимательских сетей. - СПб., 2004. - 256 с.

3. Granovetter M. Business Groups // The Handbook of Economic Sociology. N.Y., 1994. - P. 461-466. 
4. Катькало В.С. Межфирменные сети: проблематика исследований новой организационной категории в 1980-90-е гг. // Вестник СПбГУ. Сер. 5. Экономика. - 1999. - Вып. 2. - С. 21-38.

5. Elfring T., Hulsink W. Networks in entrepreneurship: The case of hightechnology firms // Small Business Economics. - 2001. - Vol. 21 (4). - P. 409-422.

6. Davidsson P., Honig B. The role of social and human capital among nascent Entrepreneurs // Journal of Business Venturing. - 2003. - Vol. 18(3). - P. 301-331.

7. Sanders J., Nee V. Immigrant self-employment: The family as social capital and the value of human capital // American Sociological Review. - 1996. Vol. 61 (2). - P. 231-226.

8. Светуньков М.Г., Волков А.В. Методы оценки предпринимательских сетей // Казанский экономический вестник. - 2013. - № 6(8). - С. 79-84.

9. Светуньков М.Г., Волков А.В. Формы контракции хозяйствующих субъектов в теории сегментной конкуренции // Известия Санкт-Петербургского государственного университета экономики и финансов. - 2010. № 1. - С. 7-13.

10. Ойстин О. Теория графов. - М.: Наука, 1980. - 336 с.

11. Варзакова А. Сетевые отношения между конкурентами (на примере рынка радиорекламы Москвы) // Экономическая социология. - 2006. - Т. 7, № 4. - C. 79-96.

12. Freeman L. Centrality in social networks, conceptual clarifications // Soc. Networks. - 1979. - Vol. 1. - P. 215-236.

13. Burt R. Models of network structure // Annu. Rev. Sociol. - 1980. Vol. 6. - P. 79-141.

14. Виханский О.С., Наумов А.И. Менеджмент: учеб. - М.: Гардарики, 2003. $-528 \mathrm{c}$.

15. Tsitsiashvili G.Sh., Osipova M.A., Losev A.S. Asymptotic analysis of few nodes failure in oriented random graph // Reliability: Theory and Applications. 2015. - Vol. 10, № 2 (37). - P. 27-29.

\section{References}

1. Radaev V.V. Rynok perepletenie sotsial'nykh setei [Market as an intertwining of social networks]. Rossiiskii zhurnal menedzhmenta, 2008, no. 2, vol. 6, pp 47-54.

2. Asaul A.N., Skumatov E.G., Lokteeva G.E. Metodologicheskie aspekty formirovaniia i razvitiia predprinimatel'skikh setei [Methodological aspects of business networks formation and development]. Saint-Petersburg, 2004, 256 p.

3. Granovetter M. Business Groups. The Handbook of economic sociology. NewYork, 1994, pp. 461-466. 
4. Kat'kalo V.S. Mezhfirmennye seti: problematika issledovanii novoi organizatsionnoi kategorii v 1980-90-e gg. [Inter-firm networks: problems of studying a new organizational category in 1980-90s.]. Vestnik SPbGU. Ser. 5. Ekonomika, 1999, issue 2, pp. 21-38.

5. Elfring T., Hulsink W. Networks in entrepreneurship: The case of hightechnology firms. Small Business Economics, 2001, no. 4, vol. 21, pp. 409-422.

6. Davidsson P., Honig B. The role of social and human capital among nascent Entrepreneurs. Journal of Business Venturing, 2003, no. 3, vol. 18, pp. 301-331.

7. Sanders J., Nee V. Immigrant self-employment: The family as social capital and the value of human capital. American Sociological Review, 1996, no. 2, vol. 61, pp. 231-226.

8. Svetun'kov M.G., Volkov A.V. Metody otsenki predprinimatel'skikh setei [Methods for entrepreneurial networks assessment]. Kazanskii ekonomicheskii vestnik, 2013, no. 6, vol. 8, pp. 79-84.

9. Svetun'kov M.G., Volkov A.V. Formy kontraktsii khoziaistvuiushchikh sub"ektov v teorii segmentnoi konkurentsii [Forms of economic entities contraction in the theory of segment competition]. Izvestiia Sankt-Peterburgskogo gosudarstvennogo universiteta ekonomiki i finansov, 2010, no. 1, pp. 7-13.

10. Oistin O. Teoriia grafov [Graph theory]. Moscow, Nauka Publ., 1980, 336 p.

11. Varzakova A. Setevye otnosheniia mezhdu konkurentami (na primere rynka radioreklamy Moskvy) [Network relations between competitors (through the example of Moscow radio advertising market)]. Ekonomicheskaia sotsiologiia, 2006, no. 4, vol. 7, pp. 79-96.

12. Freeman L. Centrality in social networks, conceptual clarifications. Soc. Networks, 1979, vol. 1, pp. 215-236.

13. Burt R. Models of network structure. Annu. Rev. Sociol., 1980, vol. 6, pp. $79-141$.

14. Vikhanskii O.S., Naumov A.I. Menedzhment [Management]. Moscow, Gardariki Publ., 2003, 528 p.

15. Tsitsiashvili G.Sh., Osipova M.A., Losev A.S. Asymptotic analysis of few nodes failure in oriented random graph. Reliability: Theory and Applications, 2015 , no. 2 , vol. 10 , issue 37 , pp. $27-29$.

Оригинальность статьи - $86 \%$

Получено 11.11.2016 


\section{A.S. Losev}

\section{BUSINESS NETWORKS ASSESSMENT}

\section{AT THE REGIONAL LEVEL}

The article considers the indicators of business networks developed in the works of M.G. Svetunkov and A.V. Volkov. The paper analyzes the methodology of modern mathematical algorithms application for network data processing, as well as business networks performance evaluation in limit and intermediate stages. Among the key indicators M.G. Svetunkov and A.V. Volkov define the network's size, communication density, heterogeneity, ranking, degree of centralization, as well as the degree of structural mobility and proximity to the network-forming element. The number of business network assessment indicators goes beyond this list and thus can be extended depending on the business dimension. The application of modern mathematical methods of business networks specifications allows proceeding from descriptive to a detailed analysis, which is indicative of a more qualitative study of the network's organization and functioning.

Keywords: business network, regional structure, network assessment, network indicators.

Aleksandr S. Losev - Candidate of Physical and Mathematical Sciences, Senior Researcher, Institute of Applied Mathematics of Russian Academy of Sciences, e-mail: A.S.Losev@yandex.ru. 